\title{
Disseminated cryptococcosis and fluconazole resistant oral candidiasis in a patient with acquired immunodeficiency syndrome (AIDS)
}

\author{
Rajendra J. Kothavade ${ }^{1}$, Chetan M. Oberai ${ }^{2}$, Arvind G. Valand ${ }^{3}$, Mehroo H. Panthaki ${ }^{4}$ \\ ${ }^{1}$ Microbiology Laboratory WQA, EPCOR, 10065, Edmonton, AB, T5J 3B1, Canada \\ ${ }^{2}$ Department of Dermatology, Sir J.J. Group of Hospitals and Grant Medical College, Mumbai, India \\ ${ }^{3}$ Department of Pathology, Sir J.J. Group of Hospitals and Grant Medical College, Mumbai, India \\ ${ }^{4}$ Department of Immunocytobiology, Sir H.N. Hospital and Medical Research Centre, Mumbai, India
}

\begin{abstract}
Disseminated cryptococcosis and recurrent oral candidiasis was presented in a-heterosexual AIDS patient. Candida tropicalis (C.tropicalis) was isolated from the oral pseudomembranous plaques and Cryptococcus neoformans (C. neoformans) was isolated from maculopapular lesions on body parts (face, hands and chest) and body fluids (urine, expectorated sputum, and cerebrospinal fluid). In vitro drug susceptibility testing on the yeast isolates demonstrated resistance to fluconazole acquired by $C$. tropicalis which was a suggestive possible root cause of recurrent oral candidiasis in this patient.
\end{abstract}

Key words: HIV; AIDS; cryptococcosis; candidiasis; immunocompromised; fluconazole

J Infect Dev Ctries 2010; 4(10):674-678.

(Received 5 December 2009 - Accepted 28 June 2010)

Copyright (C) 2010 Kothavade et al. This is an open-access article distributed under the Creative Commons Attribution License, which permits unrestricted use, distribution, and reproduction in any medium, provided the original work is properly cited.

\section{Case Report}

A 34-year-old heterosexual HIV-I positive male developed mucopurulent productive cough, recurrent oral plaques, occasional syncope, and neurological symptoms that included headache and dizziness. Physical examination revealed discrete erythematous maculopapular lesions on his face (Figure 1), neck, chest, and both hands. There were not any significant enlargement of the cervical lymph nodes, and oral examination revealed pseudo-membranous plaques (Figure 1). He was previously treated with antifungal drugs (fluconazole and amphotericin-B), primary anti-tuberculous drugs (isoniazid, rifampin, ethambutol, and streptomycin), and an antigiardial drug (tinidazole) for giardiasis, caused by, Giardia lamblia. The CD4+ lymphocyte count for this patient was 40 cells/ $\mu$ with a CD4+/CD8+ ratio of 1:72. Despite a history of multiple unprotected sexual exposures, the patient tested negative for venereal disease.

The skin biopsies and body fluids, such as, cerebrospinal fluid (CSF), urine, and mucopurulent expectorated sputum, showed encapsulated yeasts in India-ink-wet-mount preparation. The cultures for acid-fast bacilli (AFB) on Lowenstein-Jenson and non-selective Middle-brook 7H12 agar media were negative. Periodic-acid-Schiff and Grocott-Gomorimethylamine-silver-stained smears were negative for Pneumocystis carinii. Serum and CSF tested positive for capsular Cryptococcal polysaccharide antigen using the latex agglutination test with a titer of $1: 1015$. Skin sections revealed gelatinous troma (Figure 2) filled with numerous encapsulated yeast cells (Figure 3). Biopsied specimens of skin and other body fluids (CSF, urine, and sputum) yielded the growth of $C$. neoformans on Sabouraud's dextrose agar (SDA) medium. The resultant mucoid-creamcolored colonies were negative for germ tube and positive for urease test. Colonies failed to grow on Cyclohexamide-supplemented SDA. Colonic growth at $37^{\circ} \mathrm{C}$ on plain SDA was weakly positive. Microscopic examination of the Gram-stain preparation from a portion of scraped oral lesions showed Gram-positive yeasts and pseudo-hyphal forms. The remaining portion the scraped oral lesions were inoculated on SDA which then showed a typical growth of $C$. tropicals. Identification of $C$. tropicalis was further confirmed by the germ tube test; morphological characteristics were determined on cornmeal tween-80-agar and Vitek-32 and API 20C 
AUX identification systems (bioMérieux, Craponne, France). Drug susceptibility testing to fluconazole, ketoconazole, clotrimazole, posaconazole, and amphotericin-B was performed using the CLSI micro-dilution method [1]. The in-vitro drug susceptibility study revealed fluconazole resistance to $C$. tropicalis only; whereas $C$. neoformans were found to be susceptible to all antifungal agents. Lab investigations for further clinical management could not be followed as the patient died on the third day of his hospitalization.

\section{Discussion}

The two species of genus Cryptococcus ( $C$. neoformans and $C$. gatti) are pathogenic and responsible for the life-threatening infections in immunocompromised patients, particularly those that are diagnosed with HIV/AIDS [2-3]. C. albicans and other species of Candida, such as, $C$. tropicalis, are responsible for causing oral and systemic candidiasis in immunocompromised patients. Succeeding infection occurs due to high to moderately virulent strains of Candida against the intensity of the immune response generated by the host cells. Nonpathogenic Candida may colonize in immunocompromised hosts, such as, neutropenic and HIV-AIDS patients. The colonization of avirulent Candida species does occur due to poor cell mediated immune response, mounted by the compromised hosts [4].

Figure 1: Erythematous maculopapular lesions on the face and curdy coating on the tongue (oral candidiasis)

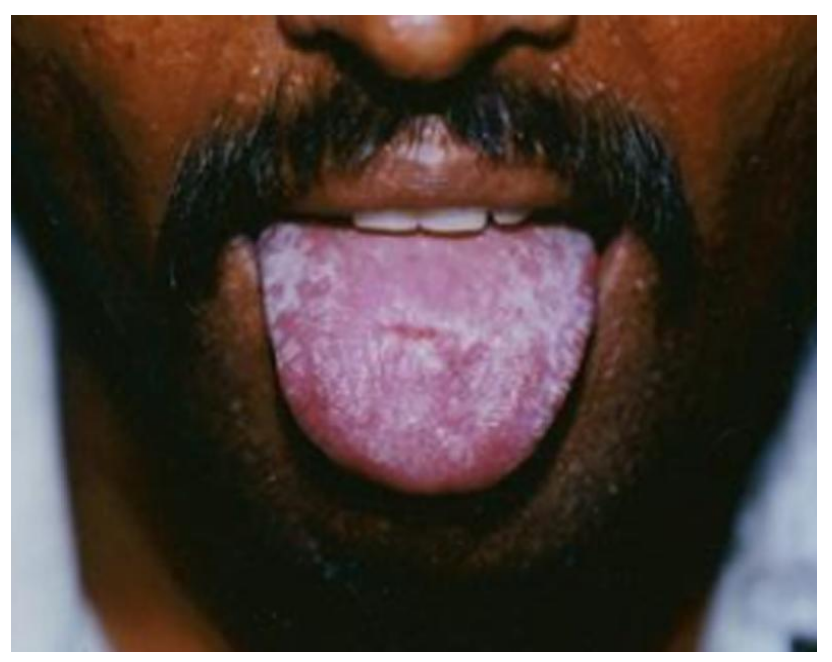

Figure 2: Histopathology of a skin biopsy from erythematous maculopapular lesions (tissue sections stained with Hematoxyllin and Eosin) shows (100x) a gelatinous stroma - (no inflammatory or granulomatous reactions).

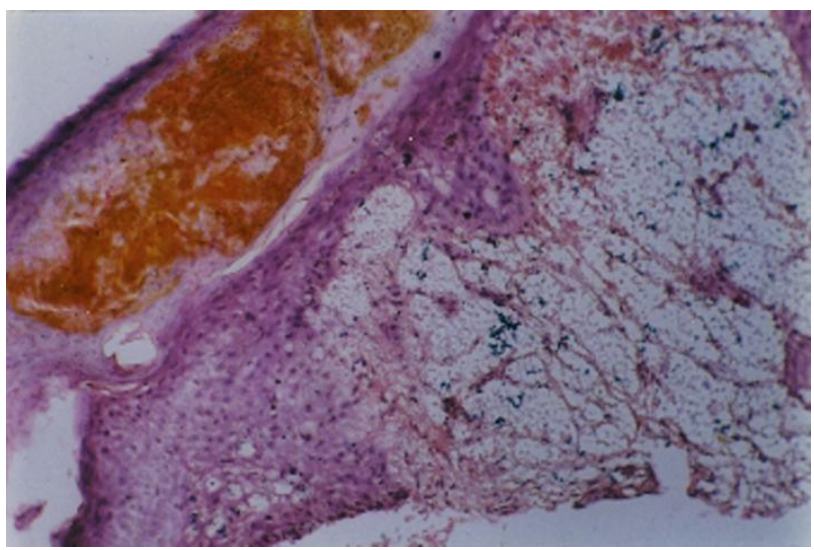

Development of Cryptococcal infection is determined by a cell-mediated immune response against an encapsulated yeast population and its virulence. Immunocompetent patients with cryptococcosis usually demonstrate granulomatous inflammatory reactions (infected sites) to encapsulated yeasts or antigens [5]. However, in this patient, there were no granulomatous reactions or inflammatory responses seen in any tissue sections; instead, there were extensive disseminated gelatinous-appearing lesions and large number of encapsulated yeasts. Comparable findings are seen in other full-blown AIDS patients as well $[6,7,8]$.

In this case study, the CD4+ cell count was an ideal marker to assess the immunological status of the patient because from the $\mathrm{T}$ lymphocyte population $\mathrm{CD} 4+$ and $\mathrm{CD} 8+$ cells are the key elements in mounting immune response against pathogenic organisms [9]. However, it has been observed from the in-vitro studies on human $\mathrm{T}$ cells that CD4+ plays a significant role in mediating CD8+ proliferation against $C$. neoformans [10]. The HIV infected patients are more prone to Cryptococcal infection when their CD4+ counts drop below 50-100 cell/mm [11,12]. In vitro studies on human $\mathrm{T}$ cells, particularly $\mathrm{CD} 4+$ and $\mathrm{CD} 8+$ cells against $C$. neoformans, have demonstrated interleukin and transferrin receptor expression with subsequent proliferative response [10]. The production of IFNgamma from CD8+ cells have been found in inactivating encapsulated yeasts of $C$. neoformans within macrophages [13]. Hence CD8+ cells in immune response may be functioning independent of 
CD4+ cells. Some experimental studies suggest, that appropriate CD4+ signaling could allow CD8+ cells to respond against $C$. neoforamans. Further studies are required in human $\mathrm{T}$ cells to explore the potential role of interleukin-2 and IFN-gamma in resolving the ambiguity of CD4+-CD8+ linked response against $C$. neoformans infection. Inadequate $\mathrm{T}$ cell (CD4+/CD8+) response against $C$. neoformans infection leads to the death of patients suffering from AIDS [14,10]. C. neoformans is the most common fatal mycosis in AIDS patients, but is less common in non-cell-mediated immunodeficiency $[15,16]$, which suggest that CMI is an important immune mechanism in the clearance of $C$. neoformans.

Figure 3: Histopathology of Skin biopsy (Erythematous maculopapular lesions): tissue sections stained with Periodic acid-schiff's stain under 400x shows intensely pinked muco-polysaccharide capsule of $C$. neoformans in gelatinous stroma.

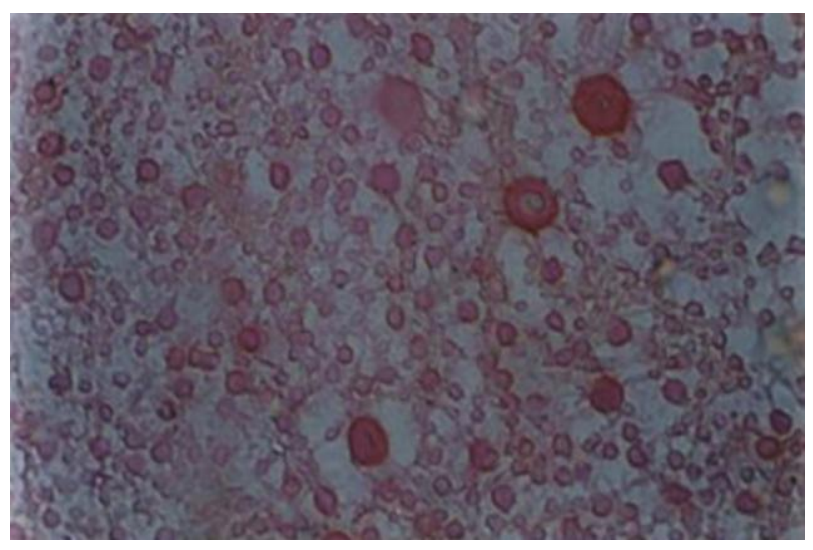

In the present case report, it appears that the low CD4+ lymphocyte count supports the opportunistic condition to establish mixed fungal infections caused by $C$. neoformans and $C$. tropicalis. Unlike $C$. albicans, which can be occasionally found as a commensal, $C$. tropicalis has almost always been associated with the development of fungal infections in immunocompromised patients [17]. Some reports from India suggests that $C$. tropicalis is the most prevalent yeast species responsible for causing infection in HIV/AIDS patients [18]. However, in the present case study, fluconazole-resistant $C$. tropicalis was isolated from the oral-pseudomembranous lesions, which was an uncommon finding in the Indian subcontinent. Such observations might have been overlooked due to a lack of drug susceptibility studies or tracking of recurrent Candida infections in immunocompromised or immunocompetent patients in India.
The extensive use of fluconazole in the clinical management of AIDS and in other high-risk patients could be the basic cause of increasing prevalence of Candida species other than $C$. albicans. Among these species $C$. tropicalis is predominant in some parts of the world [19,20,21,22]. As a result, $C$. tropicalis has been emerging in both immunocompromised and immuno-competent patients; hence, it has been suggested that the use of fluconazole in a high-risk group of patients should be avoided [19]. Irrespective of host immune response and other predisposing factors, a pathogenicity of $C$. tropicalis, might be another contributing factor for its mucosal colonization in the buccal cavity. Similarly, colonization in the gastrointestinal tract can lead to the penetration of sub-mucosal layers. Some clinical studies have shown that $C$. tropicalis is more virulent than $C$. albicans in neutropenic and non-neutropenic patients [23,24]. The purified tropiase from $C$. tropicalis (a novel acid proteinase) demonstrated hemorrhagic activity and its ability to increase capillary permeability [25]. In-spite of the recurrent infections due to $C$. tropicalis in AIDS patients, there was no evidence of its dissemination. However, dissemination did occur due to encapsulated yeasts of $C$. neoformans. The dominant mechanism of $C$. neoformans over $C$. tropicalis in immunocompromised patients is still not clearly understood.

Antifungal drug intolerance is certainly a major challenge in the clinical management of AIDS and other neutropenic and non-neutropenic patients (with several underlying subclinical conditions) [24,26]. An interesting clinico-mycological scenario in this report is that $C$. neoformans isolates were susceptible to fluconazole; however, $C$. tropicalis was found to be resistant. Such clinico-mycological aspects must be carefully considered before switching to any antifungal therapeutic options. In addition, $C$. tropicalis has often been reported as resistant to fluconazole [27]. The plausible mechanisms of fluconazole resistance have been extensively studied in the strains of $C$. albicans isolated from patients with HIV-AIDS-related oropharyngeal candidiasis [4]. Based on the literature survey, we believe that this could be the first case report from India presented with disseminated cryptococcosis and recurrent oral candidiasis due to fluconazole-resistant C. tropicalis.

Development of fluconazole resistance in $C$. tropicalis could be due to several mechanisms. Nevertheless, some plausible mechanisms [28] could 
include, escalation of emanation (efflux) of the azole compounds due to (i) over-expression of efflux pumps, such as the adenine tri-phosphate binding cassette (ABC) transporter and major facilitator superfamily (MFS) membrane transporters; (ii) upregulation of the ERG11 gene, coding for the azole target lanosterol $14(\alpha)$-demethylase; (iii) ERG11 sequence related point mutation and (iv) decreased affinity of azoles for their target-induced azole resistance. The acquired azole resistance could be the result of either one of these mechanisms or coupled with other steps in a harmonized manner [29]. In general, azole resistance in $C$. tropicalis suggests that over-expression of CtERg11, associated with missense mutation in this gene, and seemed to be responsible for acquired azole resistance.

The intracranial pressure due to the heavy load of encapsulated yeasts and extensive colonization of fluconazole-resistant $C$. tropicalis (or intolerance to antifungal agents) could be the major cause of the death of this patient. Epidemiological studies suggest that, increasing prevalence of $C$. tropicalis related to candidiasis in immunocompromised patients is due to the underlying subclinical conditions and subsequent antifungal agents associated toxicity; in addition to the genetic relatedness of the Candida species [30,31,32]. Although, morbidity and mortality rates due to infections caused by $C$. tropicalis have been reported more often, than those attributed to $C$. albicans [33]; it would be worth comparing this scenario in the cases of AIDS with disseminated cryptococcosis.

Comparing the susceptibility patterns of oral isolates during the highly active anti-retroviral therapy (HAART) era with those from 1994, suggest that: most cases of HIV-associated oropharyngeal candidiasis observed during the HAART era were caused by azole-susceptible strains. However, the reversion of isolates from azole-resistant to azolesusceptible was related to strain substitution [34]. Ever since the introduction of HAART, incidences of opportunistic infections such as, cerebral toxoplasmosis, Pneumocystis carinii pneumonia and others have been declining. However, the prevalence of Candidial esophegitis remained unchanged [34]. These findings suggests that HAART does not play a significant role in minimizing any fluconazole drug resistance in Candida species and still remains a challenging problem for its long-term therapeutic application in HIV-infected or immunocompromised patients.

\section{Conclusion}

The major gap in the pathogenicity of $C$. neoformans is the limited understanding of its dominant mechanism over $C$. tropicalis in AIDS patients. Secondly, the interlinked cellular mechanisms between CD4+ and CD8+ cells against C. neoformans infection remain unclear. Hence, some additional studies need to be performed on the expression of IL-2 receptors and IFN-gamma production by human $\mathrm{T}$ cells ( $\mathrm{T}$ cells - derived from the various clinical stages of HIV infection; may be from sero-rectaive to full blown AIDS stage).

Although, fluconazole is still the first line of treatment in a number of cases of invasive candidiasis, its therapeutic application should be restricted to selected high-risk patients in order to minimize the risk of emergence of azole-resistant strains of Candida. Consistent use of standard, rapid and reliable methods for species identification in conjunction with drug susceptibility testing is advocated; particularly, for the cases of recurrent candidiasis. This approach would allow systematic tracking of emerging Candida species, other than $C$. albicans in various clinical conditions. Additionally, its application in monitoring drug-resistant strains or changing patterns of Candida species, other than $C$. albicans in clinical settings may support clinicians in determining appropriate therapeutic agents for effective clinical management. The therapeutic use of HAART, in combination with granulocyte colonystimulating factor (G-CSF) cytokine in HIV-infected patients may be useful in the management of concomitant infections caused by fluconazoleresistant strains of $C$. tropicalis.

\section{References}

1. Villanova PA (1997) National Committee for Clinical Laboratory Standards. Reference Method for Broth Dilution Antifungal Susceptibility Testing of Yeasts. Approved Standard M27-A.

2. Kwon-Chung KJ and Bennett JE (1984) Epidemiologic differences between the two varieties of Cryptococcus neoformans. Am J Epidemiol 120: 123-130.

3. Kwon-Chung KJ and Bennett JE (1984) High prevalence of Cryptococcus neoformans var. gattii in tropical and subtropical regions. Zentbl Bakteriol Mikrobiol Hyg A 257: 213-218.

4. Franz R, Kelly SL, Lamb DC, Kelly DE (1998) Multiple molecular mechanisms contribute to a stepwise development of fluconazole resistance in clinical Candida albicans strains. Antimicrob Agents Chemother 42: 3065-3072.

5. Barret JT (1988) Textbook of immunology. An introduction to immunochemistry and immunobiology. Fifth edition. The C.V. Mosby Company, St. Louis - Washington, D.C. Toronto: pp. 121-124. 
6. Durden FM and Elewski B (1994) Cutaneous involvement with Cryptococcus neoformans in AIDS Am Acad Dermatol 30: 844-848.

7. Dinato SL, Dinato MM, Nakanishi CP, Almeida JR, Romiti $N$ (2006) Disseminated cutaneous cryptococcosis in a patient with AIDS 48: 353-358.

8. Murakawa GJ, Mauro TM, Egbert B (1995) Disseminated cutaneous Cryptococcus clinically mimicking basal cell carcinoma Dermatol Surg 2: 992-993.

9. Jiang $\mathrm{H}$ and Chess $\mathrm{L}$ (2004) An integrated view of suppressor T cell subsets in immunoregulation. J Clin Invest 114: 1198-1208.

10. Syme RM, Wood CJ, Wong H, Mody CH (1997) Both CD4+ and CD8+ human lymphocytes are activated and proliferate in response to Cryptococcus neoformans. Immunology 92: 194-200.

11. Crowe SM, Carlin JB, Stewart KI, Lucas CR, Hoy JF (1991) Predictive value of

CD4 lymphocyte numbers for the development of opportunistic infections and malignancies in HIV-infected persons. $\mathbf{J}$ Acquir Immune Defic Syndr 4: 770-776.

12. Bicanic T, Harrison T, Niepieklo A, Dyakopu N, Meintjes G (2006) Symptomatic relapse of HIV-associated cryptococcal meningitis after initial fluconazole monotherapy: the role of fluconazole resistance and immune reconstitution. Clin Infect Dis 43: 1069-1073.

13. Lindell DM, Moore TA, McDonald RA, Toews GB, Huffnagle GB (2005) Generation of antifungal effector $\mathrm{CD} 8+\mathrm{T}$ cells in the absence of CD4+ $\mathrm{T}$ cells during Cryptococcus neoformans infection. J Immunol 174: 79207928.

14. Luigina Romani (2004) Immunity to fungal infections. Nature Reviews Immunology 4: 11-24.

15. Eng RH, Bishburg E, Smith SM (1986) Cryptococcal infection in patients with acquired immune deficiency syndrome. Am J Med 81: 19-23.

16. Dismukes WE (1988) Cryptococcal meningitis in patients with AIDS. J Infect Dis 157: 624.

17. Wingard JR, Merz WG, Saral R (1979) Candida tropicalis: a major pathogen in immunocompromised patients. Ann Intern Med 91: 539-543.

18. Mathews MS, Samuel PR Suresh M (2001) Emergence of Candida tropicalis as the major cause of fungaemia in India. Mycoses 44: 278-280.

19. Charlier C, Hart E, Lefort A, Ribaud P, Dromer F, Denning DW, Lortholary O (2006) Fuluconazole for the management of invasive candidiasis: where do we stand after 15 years? J Antimicrob Chemother 57: 384-410.

20. Tortorano AM, Rigoni AL, Biraghi E, Prigitano A, Viviani MA and the FIMUA-ECMM Candidaemia Study Group (2003) The European Confederation of Medical Mycology (ECMM) survey of candidaemia in Italy: antifungal susceptibility patterns of 261 non-albicans Candida isolates from blood. J Antimicrob Chemother 52: 679-682.

21. Yang YL, Ho YA, Cheng HH, Ho M, Lo HJ (2004) Susceptibilities of Candida species to amphotericin B and fluconazole: the emergence of fluconazole resistance in Candida tropicalis. Infect Control Hosp Epidemiol 25: 6064.

22. Myoken Y, Kyo T, Fujihara M, Sugat T, Mikami Y (2004) Clinical significance of breakthrough fungemia caused by azole-resistant Candida tropicalis inpatients with hematologic malignancies. Haematologica 89: 378-80.

23. Stone HH, Kolb LD, Cume CA, Geheber CE, Cuzzeli JZ (1974) Candida sepsis: pathogenesis and principles of treatment. Ann Surg 179: 697-711.

24. Walsh TJ and Merz WG (1986) Pathologic features in the human alimentary tract associated with invasiveness of Candida tropicalis. Am J Clin Pathol 85: 498-502.

25. Yoshiyuki O, Naomasa I, Toshiaki N (2007) Isolation and Characterization of a Novel Acid Proteinase, Tropiase from Candida tropicalis IFO 0589. Jpn J Med Mycol 48: 19-25.

26. Graybill JH, Najvar LK, Holmberg JD, Luther MF (1995) Fluconazole, D0870, and flucytosine treatment of disseminated Candida tropicalis infections in mice. Antimicrob Agents Chemother 39: 924-929.

27. Sanglard D and Odds FC (2002) Resistance of Candida species to antifungal agents: molecular mechanisms and clinical consequences. Lancet Infect Dis 2: 73-85.

28. Morschha"user J (2002) The genetic basis of fluconazole resistance development in Candida albicans. Biochim Biophys Acta 1587: 240-248.

29. Ellis M (2001) Invasive fungal infections: evolving challenges for diagnosis and therapeutics. Mol Immunol 38: 947-957.

30. Kao AS, Brandt ME, Pruitt WR, Conn LA, Perkins BA, Stephens DS, Baughman WS, Reingold AL, Rothrock GA, Pfaller MA, Pinner RW, Hajjeh RA (1999) The epidemiology of candidemia in two United States cities: results of a population-based active surveillance. Clin Infect Dis 29: 1164-1170.

31. Kontoyiannis DP, Vaziri I, Hanna HA, Boktour M, Thornby J, Hachem R, Bodey GP, Raad II (2001) Risk factors for Candida tropicalis fungemia in patients with cancer. Clin Infect Dis 33: 1676-1681.

32. Flynn PM, Marina NM, Rivera GK., Hughes WT (1993) Candida tropicalis infections in children with leukemia. Leuk Lymphoma 10: 369-376.

33. Tacconelli E, Bertagnolio, S, Posteraro B, Tumbarello M, Boccia S, Fadda G, Cauda R (2002) Azole susceptibility patterns and genetic relationship among oral Candida strains isolated in the era of highly active antiretroviral therapy. J Acquir Immune Defic Syndr 31: 38-44.

34. Traeder C, Kowoll S, Arastéh K (2008) Candida infection in HIV positive patients 1985-2007. Mycoses 5: 58-61.

\section{Corresponding author}

Dr. R. J. Kothavade

Microbiology Laboratory -WQA

EPCOR

10065, Jasper Ave

Edmonton, AB, T5J 3B1

Canada

Tel/Fax (780) 412-7617

Email: rkothava@epcor.ca

Conflict of interests: No conflict of interests is declared. 